\title{
Myosin1f-mediated neutrophil migration contributes to acute neuroinflammation and brain injury after stroke in mice
}

\author{
Yan Wang ${ }^{1}$, Haojie Jin ${ }^{2}$, Weifang Wang $^{3}$, Feng Wang ${ }^{3^{*}}$ and Heng Zhao ${ }^{1 *}$
}

\begin{abstract}
Background: During the acute stroke phase, neutrophils from the peripheral blood are first to arrive in the ischemic brain, which then attracts other immune cells that exacerbate neuroinflammation in the ischemic tissue. Myosin $1 \mathrm{f}$ was reported to specifically mediate neutrophil migration in the peripheral tissues, but whether it plays a critical role in the neuroinflammatory response after ischemic stroke remains unknown. In this study, we aim to test the hypothesis that myosin 1f-mediated neutrophil migration is critical in acute neuroinflammation induced by ischemic stroke.
\end{abstract}

Methods: Myosin $1 f^{-/-}$and wild type (WT) mice were subjected to transient middle cerebral artery occlusion (MCAO). To determine which cells determine myosin $1 f^{\prime}$ s transmigration ability, bone marrow transplantation, neutrophil depletion, and adoptive neutrophil transfer were performed. The myosin $1 \mathrm{f}$ RNA level was assessed in peripheral neutrophils by reverse transcription polymerase chain reaction (RT-PCR) at 1 day and 3 days after stroke. The infiltrating neutrophils were quantified by immunofluorescence staining and FACS at $72 \mathrm{~h}$ after reperfusion.

Results: The myosin $1 \mathrm{f}^{-/-}$mice had significantly smaller infarctions than the myosin $1 \mathrm{f}^{+/+}$mice. Bone marrow transplantation from myosin $1 f^{-1-}$ mice to recipient mice also had smaller infarctions compared to animals receiving bone marrow from myosin $1 f^{+/+}$mice. By performing neutrophil depletion and adoptive transfer, we confirmed that myosin $1 f$ acts mainly in circulating neutrophils. RT-PCR showed that myosin $1 f$ gene expression was increased in the circulating blood neutrophils at 3 days after ischemia. The confocal immunostaining and FACS results confirmed that fewer neutrophils infiltrated into the ischemic brain in myosin $1 f^{-1-}$ mice compared to WT mice.

Conclusions: Myosin 1f determines neutrophil migration into the ischemic hemisphere, which directly affects stroke outcome.

Keywords: Ischemic stroke, Neuroinflammation, Myosin1f, Neutrophils, Migration

\section{Introduction}

Ischemic stroke is the most common acute cerebrovascular disease worldwide and accounts for approximately $80 \%$ of stroke cases [1]. However, the two major FDA-approved therapeutics, the blood clot dissolvent $\mathrm{t}-\mathrm{PA}$, and mechanical devices used to remove blood

\footnotetext{
* Correspondence: wangfeng16@sjtu.edu.cn; hzhao@stanford.edu

${ }^{3}$ Center for Microbiota and Immunological Diseases, Shanghai General Hospital, Shanghai Institute of Immunology, Shanghai Jiao Tong University School of Medicine, 280 South Chongqing Road, Building 5\#, Room 807\#, Shanghai 200025, China

'Department of Neurosurgery, Stanford University School of Medicine, 1201 Welch Road, MSLS Building, Room P306, Stanford, CA 94305, USA Full list of author information is available at the end of the article
}

clots, are only available for a small portion of stroke patients with restrictive conditions [2]. Therefore, it is necessary to study leukocyte-mediated neuroinflammatory mechanisms that modulate stroke outcomes to provide new avenues for effective therapeutics against stroke.

After a stroke, the injured neurons release inflammatory cytokines, which recruit immune cells crossing the disrupted brain-blood barrier (BBB) into the ischemic brain [3]. The circulating immune cell migration starts within a few hours of stroke onset, and neutrophils are among the first circulating leukocytes to arrive at the ischemic hemisphere. Previous studies have reported that neutrophil depletion attenuates infarction size in mouse

(c) The Author(s). 2019 Open Access This article is distributed under the terms of the Creative Commons Attribution 4.0 International License (http://creativecommons.org/licenses/by/4.0/), which permits unrestricted use, distribution, and 
models [4]. In addition, neutrophil migration also plays an essential role in stroke outcome. For instance, neutrophil migration inhibited by blocking the very-late-antigen-4 (VLA-4) reduces brain injury and behavioral impairment [5]. Clinically, diffusion-weighted MR imaging showed that the more neutrophils migrate into the ischemic hemisphere, the higher chance of larger infarct volumes in acute phase after stroke [6].

The class I myosin (myosin1) is a subgroup of the myosin superfamily and is evolutionarily conserved in both mice and human beings $[7,8]$. The fibrous myosin proteins consist of three major domains: the head, the converter, and the tail. While the tail domains enable myosin 1 to bind to cell membrane lipids, its head domains are able to bind to actin filament, which may connect with chromatin in the nuclei [9]. It contains eight class I myosin members, six of which have short-tailed forms (myosin $1 \mathrm{a}, \mathrm{b}, \mathrm{c}, \mathrm{d}, \mathrm{g}$, and $\mathrm{h}$ ), and two of which have long-tailed forms (1e and f). The short-tailed forms are involved in more specialized functions, i.e., vesicles transportation, the adaptation of hair cells in the ear [9, 10], and structure maintenance [11]; while the long-tailed forms function in cell motility and migration [8]. Myosin1f interacts with actin filaments to form an actin-myosin complex within the cytoskeleton of leukocytes to enable the transendothelial and interstitial migration of leukocytes [8]. Myosin1f is selectively expressed in the peripheral immune organs, such as the spleen, lymph nodes, and thymus [8]. Within the lymphoid tissue, only neutrophils selectively express myosin1f, while other leukocytes, such as natural killer (NK) cells, dendritic cells, and macrophages express both forms of long-tailed myosin1f and 1e [8]. A recent report shows that myosin $1 \mathrm{f}$ is specifically required for neutrophil migration in a 3D environment during the acute inflammation phase of peritonitis and lung injury in the mouse model [12]. Based on these previous studies, we aimed to test the hypothesis that myosin $1 \mathrm{f}$-mediated neutrophil migration modulates neuroinflammation and brain injury after stroke.

\section{Methods}

\section{Animals}

Male WT C57BL/6J and myosin $1 \mathrm{f}^{-1-}$ mice were purchased from The Jackson Laboratory (Bar Harbor, ME), then bred and housed at the Stanford Medical School Animal Care Facility. Experimental protocols were approved by the Stanford University Administrative Panel on Laboratory Animal Care (APLAC). Experiments were performed in accordance with the ARRIVE guidelines and the National Institutes of Health (NIH) Guidelines for the Care and Use of Laboratory Animals with local government approval [13]. A total of 98 male mice were included in this study. All of the animals were randomly assigned to different groups. Animals were excluded according to the following criteria: (1) had no neurological deficits after stroke; (2) brains had evidence of surgical subarachnoid hemorrhage; (3) the bone marrow-transplanted mice did not show $\mathrm{CD} 45.2^{+}$by FACS. For all animal studies, the surgeon did not perform the pre-treatments (including bone marrow transplantation, neutrophil depletion, and adoptive transfer) on the mice.

\section{Focal cerebral ischemia}

Anesthesia was induced by $5 \%$ isoflurane and maintained at $1-2 \%$ isoflurane throughout surgery. Body temperature was maintained at $37 \pm 0.5^{\circ} \mathrm{C}$ with a surface heating pad during the entire procedure. Focal cerebral ischemia was induced by $45 \mathrm{~min}$ transient MCA occlusion by inserting a silicone-coated 6-0 monofilament (Doccol Corp, Redlands, CA) into the left CCA to block the MCA, as we have reported previously [14]. Sham-operated mice underwent the same procedure, but without monofilament insertion. The cerebral blood flow (CBF) was detected before stroke onset, during MCA occlusion, and after reperfusion by using laser Doppler probe. The details are listed in Additional file 1: Table S1.

\section{Infarction measurement}

At $48 \mathrm{~h}$ or $72 \mathrm{~h}$ after ischemia, the mice were deeply anesthetized with isoflurane and euthanized. The brains were sliced into 5 slices with 2-mm thickness and stained in $2 \%$ 2, 3, 5-triphenyl tetrazolium chloride (TTC, ${ }^{*}$ T8877, Sigma Aldrich, St Louis, MO) for 10-15 min at $37^{\circ} \mathrm{C}$, and fixed in $4 \%$ PFA overnight. The brain infarctions were then measured using NIH ImageJ software [15]. It was normalized to the contralateral hemisphere and expressed as a ratio according to the following formula: (area of non-ischemic hemispherearea of non-ischemic tissue in the ischemic hemisphere)/area of non-ischemic hemisphere [16].

\section{Neurobehavioral examination}

Neurobehavioral tests were carried out by an investigator who was blinded to the treatment. This assay is based on a modified neurological severity score (mNSS) system to present a comprehensive neurological function assessment, including motor, sensory, balance, and reflex tests [17]. mNSS scores range from 0 to 14 , in which 0 represents normal and 14 represents the highest degree of neurological deficiency. For the motor assay, after raising the mouse by the tail, the bend and torsion of limbs were observed (score $0-3$ ). The walking posture was also checked (score $0-3$ ). For the balance test, mice were placed on a beam to see whether the mouse could keep their balance, if their limbs fell off the beam, and 
they could walk on the beam (score 0-6). For the sensory and reflex tests, pinna and corneal reflexes were examined, respectively (score $0-2$ ).

\section{Bone marrow transplant}

For the bone marrow chimera experiments, B6.SJL-Ptprca Pepcb/ BoyJ (CD45.2 ${ }^{-}$) mice (stock number 002014) were purchased from Jackson Laboratory (Bar Harbor, ME). Further details are found in our previous publication [18]. Briefly, male wild-type B6.SJL mice were subjected to irradiation $(950 \mathrm{rad})$, then $5 \times 10^{6}$ bone marrow cells from wild-type C57BL/6J $\left(\mathrm{CD} 45.2^{+}\right)$or Myosin1 $\mathrm{f}^{-/-}\left(\mathrm{CD} 45.2^{+}\right)$mice were IV injected into each irradiated recipient. Eight weeks after bone marrow transfer, peripheral blood CD45.2 expressions were detected by flow cytometry to ensure the successful bone marrow transfer.

\section{Neutrophil depletion}

For the neutrophil depletion experiment, In vivo Mab anti-mouse Ly6G (clone 1A8) (Bio X Cell, W. Lebanon, $\mathrm{NH}$ ) antibody was IP injected 1 day before and 1 day after surgery to induce neutrophil depletion $(250 \mu \mathrm{g} / \mathrm{mice})$. The neutrophil population was then monitored using FACS.

\section{Neutrophil and monocyte purification}

Neutrophils and monocytes were collected from both bone marrow and spleen. Bone marrow cells were flushed from the femora and tibia; the spleen cells were harvested from male C57BL/6J mice (myosin1f ${ }^{+/+}$) or myosin $1 \mathrm{f}^{-/-}$mice. Cells were then treated with ACK lysis buffer (Invitrogen, Carlsbad, CA) to lyse the red blood cells. After washing with PBS, cells were stained with PE-conjugated Ly6G antibody (clone 1A8) (BioLegend, San Diego, CA) on ice for $20 \mathrm{~min}$. The cells were washed again with PBS, and then labeled with magnetic anti-PE microbeads (Miltenyi Biotec, Sunnyvale, CA) for $15 \mathrm{~min}$ at $4{ }^{\circ} \mathrm{C}$. The cells were then separated using a MACS column and MACS separator, according to the manufacturer's instructions. For monocyte purification, PE-conjugated F4/80 antibody (clone BM8) (BioLegend, CA) was used as the primary antibody, followed by labeling with magnetic anti-PE beads, as indicated above.

\section{Neutrophil adoptive transfer}

B6.SJL-Ptprca Pepcb/BoyJ (CD45.2-) mice were used as the recipient mice for the adoptive neutrophil transfer experiments. Neutrophils were first depleted using anti-mouse Ly6G (clone 1A8) via IP injection $(250 \mu \mathrm{g} /$ mice). After $36 \mathrm{~h}$, purified neutrophils from myosin $1 \mathrm{f}^{-/-}$ mice $\left(\mathrm{CD} 45.2^{+}\right)$or wild-type C57BL/6J mice (myosin1f $\left.{ }^{+/+}, \mathrm{CD} 45.2^{+}\right)$were adoptively transferred via IV injection. The CD45.2 expression was monitored by fluorescence-activated cell sorting (FACS).

\section{Real-time PCR}

To evaluate the myosin $1 \mathrm{f}$ expression levels after stroke, neutrophils were first collected from whole blood in the sham control group 1 day and 3 days after stroke. Whole blood was collected into pretreated $\mathrm{K}_{2}$ EDTA tubes (BD Life Sciences, Franklin Lakes, NJ). The red blood cells were lysed with ACK lysis buffer and then selected using MACS magnetic beads (details in neutrophils purification). RNA was purified using an RNeasy Mini Kit (Qiagen, Valencia, CA). The RNA quality was measured using the Bioanalyzer. The relative expression of myosin $1 f$ were performed using the TaqMan method at the Stanford Pan facility. Myosin1f TaqMan primer (Assay ID: Mm01271176_m1) and reference gene $\beta$-actin (Assay ID: Mm00615581_s1) were purchased from Thermo Fisher Scientific (Waltham, MA). The relative ICAM-1 and MAC-1 expressions were measured using SYBR green products-based RT-PCR. The primers were synthesized from the Stanford Pan facility. The detailed primer sequences are listed in Additional file 1: Table S2.

\section{Neutrophils transmigration assay}

Neutrophils were first purified from myosin $1 \mathrm{f}^{-/-}$mice or wild-type C57BL/6J mice bone marrow using MACS magnetic beads (details in neutrophils purification). Neutrophil transmigration was analyzed using transwell plates (Corning, Tewksbury, MA). Twenty-micrometer $\mathrm{N}$-formyl-methionyl-leucyl-phenylalanine (fMLP) was added in the lower chamber, and cells were treated using fibronectin protein, as previously described $[8,12]$. After $3 \mathrm{~h}$ incubation by $5 \% \mathrm{CO} 2$ at $37^{\circ} \mathrm{C}$, the cells that migrated into the lower chamber were counted. Briefly, $100 \mu \mathrm{L}$ of trypan blue-treated cell suspension was applied to a glass hemocytometer. Using a microscope, the hemocytometer gridlines were viewed with $\mathrm{a} \times 10$ objective and the cells were counted.

\section{Immunostaining}

After a stroke, mice were transcardially perfused with PBS and fixed in $4 \%(w / v)$ paraformaldehyde. Mouse brains were cut into $30 \mu \mathrm{M}$ coronal slices using a microtome (Leica3050, Wetzlar, Germany). For staining, brain slices were washed in $0.3 \%$ Triton for 5 min and blocked with BSA at room temperature for $2 \mathrm{~h}$. After removing the blocking buffer, a 1:200 dilution of primary antibody, anti-myeloperoxidase antibody (MPO) (Abcam, Cambridge, MA) was added and incubated overnight at $4{ }^{\circ} \mathrm{C}$. To remove the primary antibody, the slices were washed three times for $15 \mathrm{~min}$ with PBS. A 1:200 dilution of Alexa 488-conjugated goat anti-mouse antibody (Invitrogen, Carlsbad, CA) was added and then incubated at room temperature for $2 \mathrm{~h}$ in the dark. The secondary antibody was removed by washing the slices three times in PBS, mounted with DAPI (Vector Laboratories, 
Burlingame, CA), and stored with a coverslip. Fluorescent images were taken by a confocal microscope (Zeiss LSM510, Jena, Germany) and analyzed using Zen software (https://www.zeiss.com/microscopy/int/products/ microscope-software/zen-lite.html).

\section{Fluorescence-activated cell sorting}

The brain immune cells were isolated, as described previously [19]. In brief, mice were deeply anesthetized with isoflurane, euthanized, and then transcardially perfused with cold PBS. The ischemic hemispheres were collected for FACS staining. For sham control, the whole brain was collected. The brain tissues were mildly homogenized on ice and centrifuged at $1800 \mathrm{rpm}$ for $5 \mathrm{~min}$. Thirty percent Percoll was then added, centrifuged twice for $4 \mathrm{~min}$, and then washed with PBS. For FACS intercellular staining, the cells rested for $2 \mathrm{~h}$ at $37^{\circ} \mathrm{C}$ in $5 \%$ $\mathrm{CO} 2$, then washed with PBS. The cells were then stained with live/dead aqua (Thermo Fisher Scientific, Waltham, MA) on ice in the dark for $10 \mathrm{~min}$, and then stained on ice for $30 \mathrm{~min}$ with FITC anti-mouse CD45 (clone 30-F11), Pacific blue anti-mouse/human CD11b (clone M1/70), and PE anti-mouse Ly6G (clone 1A8). Cells were fixed and permeabilized using intracellular flow cytometry (all the antibodies and fix buffer were purchased from BioLegend).

\section{Statistics}

Animal numbers were calculated prior to the experiments using G*Power software [20]. The comparison of the difference between two independent means with two tails was analyzed, using a priori with given $\alpha(0.05)$ and power (0.95). The estimated average mean for wild type was set to be 39 (with $\mathrm{SD} \pm 5$ ) and the difference above $25 \%$ (mean value for knock out $\sim 27$ with $\mathrm{SD} \pm 5$ ) was considered as a significant difference. The estimated number of samples for each group is five or more. All results are presented as mean \pm SD. Statistical significance of differences among groups was determined by two-tailed unpaired and paired Student's $t$ test. For experiments with one treatment and more than three groups, one-way ANOVA was applied. For experiments with more than one treatment or variants, two-way ANOVA was performed followed by Tukey's multiple comparisons test using GraphPad Prism 7.0 (GraphPad Software, La Jolla, CA. www.graphpad.com).

\section{Results}

Our data showed that myosin $1 f^{-1-}$ mice had significantly smaller infarctions compared to WT mice (Fig. 1A). As the previous study showed that myosin $1 \mathrm{f}$ is mainly expressed in the peripheral lymphoid organ but not in the brain [8], suggesting that myosin $1 \mathrm{f}$ is mainly involved in immune cells and tissues, and it is very likely that myosin $1 \mathrm{f}$ affects brain injury via circulating leukocytes. To test this, bone marrow chimeric mice were constructed (Fig. 1B), and the results show that the recipient mice receiving bone marrow cells from myosin $1 \mathrm{f}$ ${ }^{-1-}$ mice had less brain damage compared to animals receiving myosin $1 \mathrm{f}^{+/+}$bone marrow cells, suggesting that circulating leukocytes are critical for myosin $1 \mathrm{f}$ in determining stroke outcomes.

To identify whether myosin $1 \mathrm{f}$ in neutrophils directly affects stroke outcomes, we conducted a two-step experiment. First, neutrophils were depleted to test the role of neutrophils in stroke outcomes. Ly6G antibodies were IP injected into bone marrow-transplanted mice for two consecutive days, at 1 day before and 1 day after stroke to ensure the successful depletion of $\mathrm{Ly} 6 \mathrm{G}^{+}$cells (Fig. 1C; Additional file 1: Figure S1). The results show that after neutrophil depletion, the infarction sizes were both decreased and comparable between myosin $1^{-1-}$ and myosin $1 \mathrm{f}^{+/+}$mice. Second, to further confirm the role of myosin $1 \mathrm{f}$ in neutrophils, the purified neutrophils from myosin $1 \mathrm{f}^{+/+}$and myosin $1 \mathrm{f}^{-/-}$mice $\left(\mathrm{CD} 45.2^{+}\right)$were adoptively transferred to recipient mice $\left(\mathrm{CD} 45.2^{-}\right)$, respectively, whose neutrophils had been depleted by the injection of Ly6G antibodies (Fig. 1D). The infarctions were measured 2 days after the adoptive transfer based on pre-measured time courses (Additional file 1: Figure $\mathrm{S} 2$ ). Our results showed that adoptive myosin $1 \mathrm{f}^{-/-}$neutrophil transfer resulted in smaller infarction compared with myosin $1 \mathrm{f}^{+/+}$neutrophil transfer in mice (Fig. 1D).

Indeed, the RT-PCR analyses showed the peripheral circulating neutrophil myosin1f expression was increased at 3 days after ischemic stroke compared to sham control (Fig. 2A). In addition, in vitro migration assay showed that purified neutrophils from myosin $1 \mathrm{f}$ -I- mice had decreased migration ability compared to WT mice, but not in monocytes (Fig. 2B). This confirms that myosin $1 \mathrm{f}$ is necessary for peripheral neutrophil migration but not monocytes after ischemia. The immunofluorescence staining (IF) showed that stroke resulted in a significant decrease of $\mathrm{MPO}^{+}$cells in myosin1f ${ }^{-/-}$ mice (Fig. 2C). The FACS results further confirmed that both total immune cell numbers and infiltrating neutrophil numbers decreased in myosin $1 \mathrm{f}^{-/-}$mice. The number of monocyte-derived macrophages (MoDMs) also showed a reduction (Fig. 2D).

It is known that the intracellular adhesion molecule 1 (ICAM-1) and macrophage-1antigen (MAC-1) are functionally required for neutrophils and other leukocytes during adhesion and crawling [21-23], we therefore further assessed their expression levels. We used RT-PCR to measure these gene expressions in purified neutrophils at 3 days after stroke. The results show that both ICAM-1 and MAC-1 did not show a significant difference (Additional file 1: Figure S3), demonstrating that 


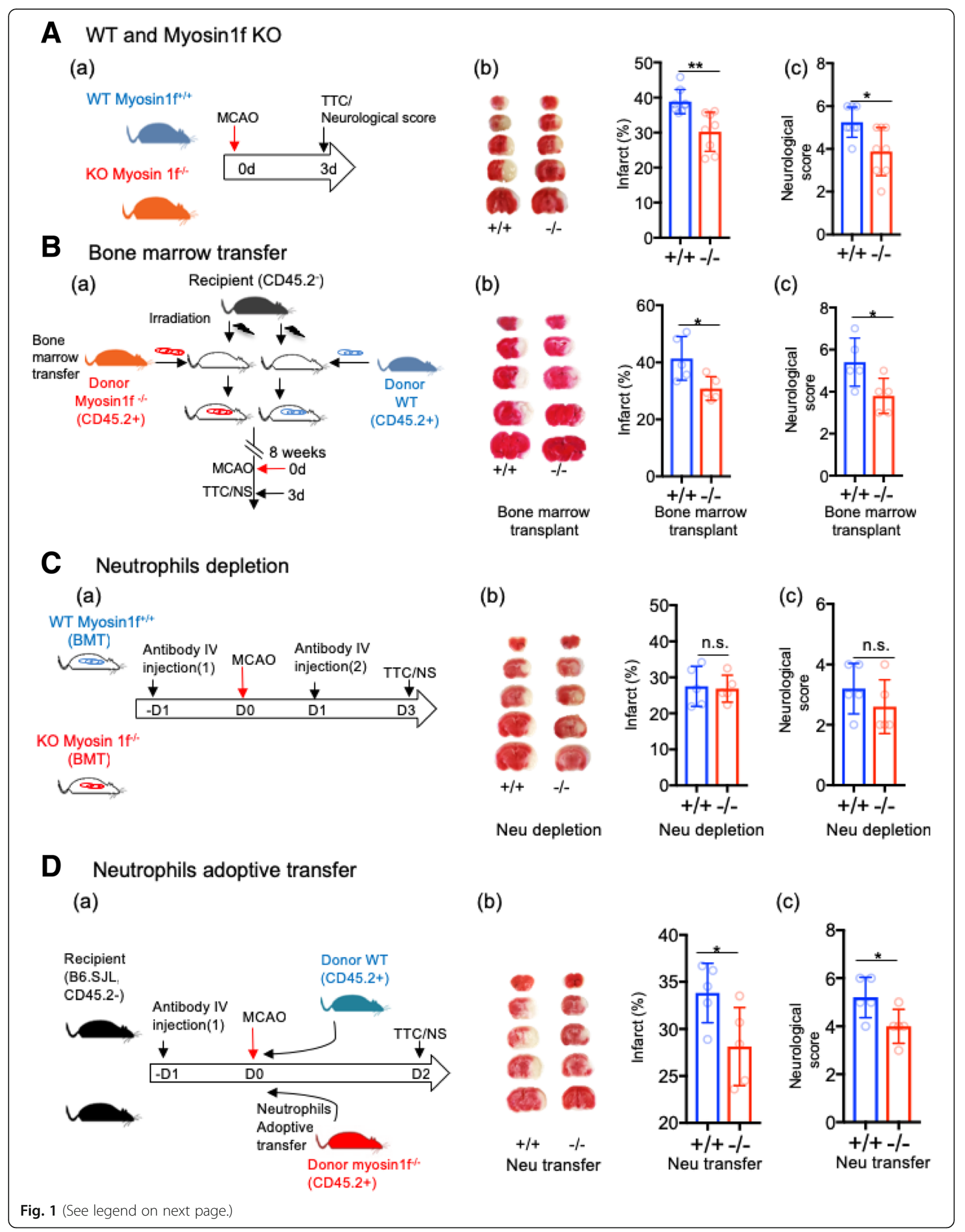




\section{(See figure on previous page.)}

Fig. 1 Myosin $1 \mathrm{f}$ affects ischemic brain injury through circulating neutrophils. A Myosin $1 \mathrm{f} \mathrm{KO}$ mice has smaller infarction. (a) The experimental procedure. The WT mice are indicated in blue; myosin1f KO mice are indicated in red. (b) Representative TTC-stained brain infarction and quantification of infarct size, which are expressed as percentages to the ischemic hemisphere $(p=0.023)$. (c) Neurological scores measured $3 d$ after stroke ( $p=0.0111, n=8$ mice/group). B Circulating leukocytes are critical for the protective effects of myosin $1 \mathrm{f}^{-/-}$against stroke. (a) Experimental procedures to study the effect of bone marrow transfer on stroke outcomes: after irradiation, recipient WT mice (CD45.2myosin $1 \mathrm{f}^{+/+}$) received bone marrow cells from WT mice $\left(\mathrm{CD} 45.2^{+}\right.$, myosin $\left.1 \mathrm{f}^{+/+}\right)$or myosin $1 \mathrm{f}^{-/-}$mice $\left(\mathrm{CD} 45.2^{+}\right.$, myosin $1 \mathrm{f}^{-/-}$, respectively. Eight weeks later, animals were subjected to MCAO; the infarction and neurological scores were assessed at 3 days after stroke. (b) Infarct sizes: representative $\Pi \mathrm{TC}$ staining and quantification of infarctions $(p=0.0263)$. (c) Neurological score. ( $p=0.0353, n=5$ mice/group). C The effects of circulating neutrophil depletion. (a) Experimental procedures: Ly6G antibodies were IV injected 1 day before and 1 day after stroke to deplete neutrophils. (b) Infarct sizes. $N=5$ mice/group. (c) Neurological scores. D Adoptive transfer of myosin $1 f^{-/-}$neutrophils attenuated brain infarction. (a) Experimental procedures to study the effects of neutrophil transfer on stroke outcomes: the recipient mice were IV injected with Ly6G antibodies for neutrophil depletion. The animals then received adoptive transfer of purified neutrophils from WT and myosin $1 f^{-1-}$ mice. Animals were euthanized 2 days later. (b) Infarct sizes ( $p=0.0409, n=5$ mice/group). (c) Neurological scores ( $p=0.0400, n=5$ mice/group)

neutrophil extravasation was not dependent on the expression level changes of these factors, but more likely related to myosin1f.

\section{Discussion}

In our current study, we showed that myosin1f $\mathrm{KO}$ specifically attenuates neutrophil migration ability, both in vitro and in vivo. Myosin $1 f^{-/-}$mice had smaller infarction compared to WT mice. Moreover, recipient mice receiving myosin $1 \mathrm{f}^{-/-}$neutrophils had better stroke outcome compared to animals receiving myosin $1 \mathrm{f}^{+/+}$ neutrophils.

Stroke-induced ischemic injury triggers a robust inflammatory response in a few hours after blood vessel occlusion. This inflammation starts with a local reaction that includes inflammatory cytokines and chemokines released from injured neurons and brain-resident microglia, followed by the recruitment of leukocytes from peripheral circulation [24, 25]. Neutrophils are rapidly mobilized from bone marrow after stroke and are the first cell type to arrive in the ischemic brain, thereby initiating an efficient innate immune response that peaks around 2 days to 3 days [26, 27]. Neutrophil infiltration is associated with increased infarction size, brain-blood barrier (BBB) disruption, and reduced neurological scores in both mice and patients [6, 28, 29]. Previous studies showed that direct neutrophil depletion using antibodies reduces the BBB breakdown, attenuates brain injury, and decreases brain inflammation in both intracerebral hemorrhage and focal cerebral ischemia mice models $[4,30]$.

Other experimental evidence shows that disrupting neutrophil infiltration also results in significant protection after stroke. There are three main steps for neutrophil infiltration including capture and rolling, adhesion and crawling, and transmigration [31]. The first two steps have been well studied [32]. For instance, blocking the very-late-antigen-4 (VLA-4) effectively inhibits neutrophil interaction with endothelial cells, and significantly decreases brain injury [5]. In addition, the neutrophil adherence antagonist, WEB2086, which inhibits the platelet-activating factor (PAF) and blocks the leukocyte adhesion to endothelial cells, showed beneficial effects during spinal cord ischemia-reperfusion in rabbits [33]. Moreover, the two molecules, intracellular adhesion molecule 1 (ICAM-1), and macrophage-1antigen (MAC-1) are functionally required for neutrophils and other leukocytes during adhesion and crawling [32]. The ICAM-1 levels are increased after stroke in both animals and patients [21,22], and blocking ICAM-1using antibodies reduces ischemic damages after transient MCAO in rats [23]. Genetic depletion of ICAM-1 also decreases infarct size and improves outcomes in a rodent model [34]. MAC-1 blocks by using neutrophil inhibitory factor (NIF) results in smaller infarction and functional outcome improvement in rats $[35,36]$. Nevertheless, the molecular mechanisms of neutrophil transmigration remain less studied compared with the first two stages of leukocyte recruitment [32].Thus, we investigated the role of myosin $1 \mathrm{f}$ in neutrophils transmigration.

A recent study showed that myosin $1 \mathrm{f}$ is specifically required for neutrophil migration in acute peritonitis and acute lung injury mouse model [12]. In line with this study, our data confirmed that myosin $1 \mathrm{f} \mathrm{KO}$ attenuates neutrophil migration into the ischemic hemisphere. The downregulation of migration ability was only found in neutrophils rather than in monocytes. One possible reason is that myosin1f is the only long-tailed isoform expressed in neutrophils, while both $1 \mathrm{e}$ and $1 \mathrm{f}$ are expressed in other cells type, including monocytes/macrophages, NK cells, and dendritic cells [8]. The two isoforms, myosin $1 \mathrm{e}$ and $1 \mathrm{f}$, are not functionally identical. Myosin1f was reported to specifically regulate neutrophil migration, but did not affect neutrophil phagocytosis [8]. For instance, myosin1e is involved in phagocytosis and phagosome closure $[37,38]$, but myosin1f is not [8]. In addition, myosin1e is required for plasma membrane tension, but myosin $1 \mathrm{f}$ is not required for cortical tension, one of the main characters of plasma membrane 

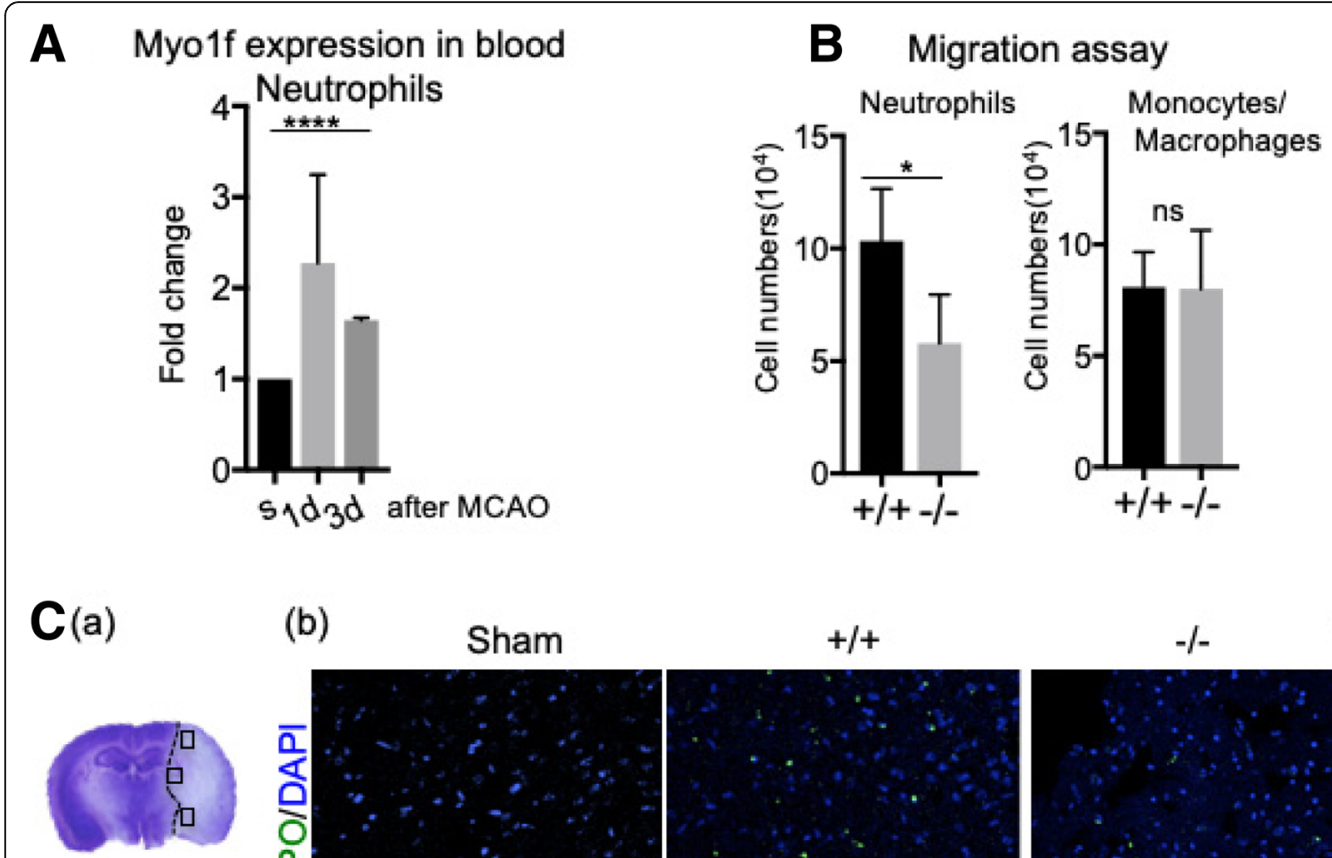

(b)
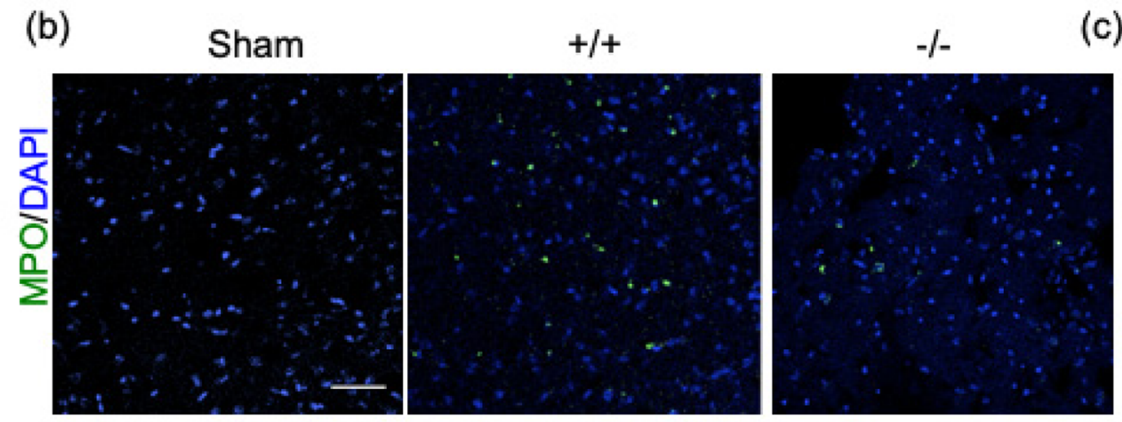

(c)

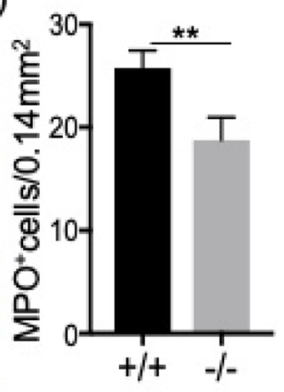

D

(a)
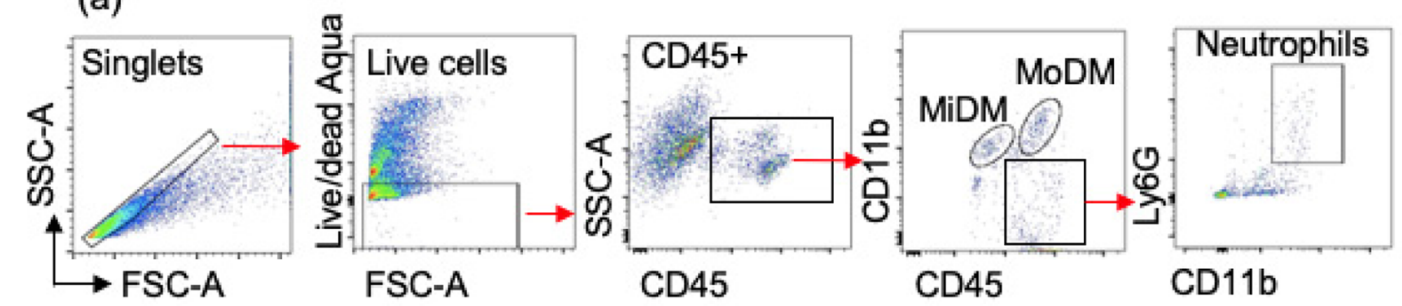

(b)

- +/+ Sham - -/- Sham $\square+/+$ MCAO - - MCAO
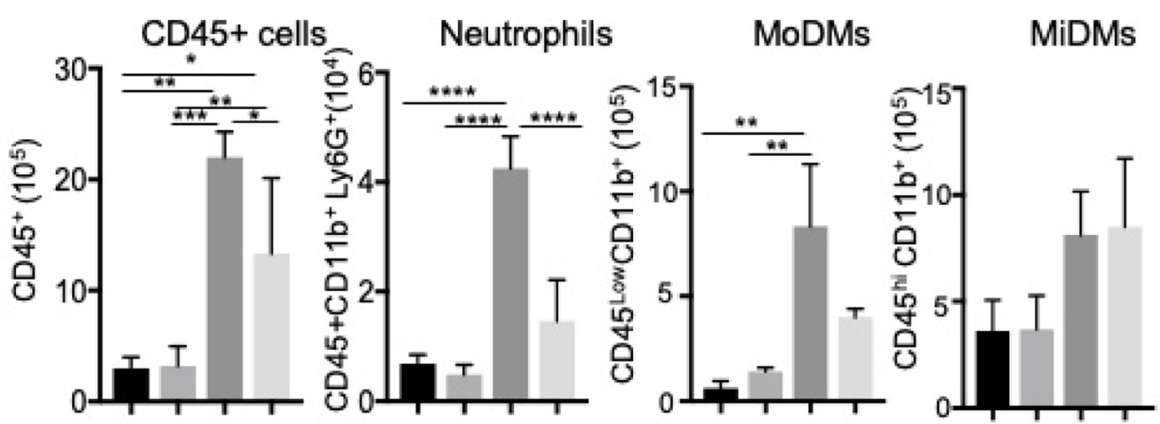

Fig. 2 (See legend on next page.) 
(See figure on previous page.)

Fig. 2 Myosin $1 f$ is required for neutrophil migration after acute ischemic stroke. A Myosin $1 f$ gene expression increased in blood neutrophils at 3 days after stroke compared to the sham control group(s), ${ }^{* * * *} p<0.0001, n=3$ mice/group; each has 3 replicates. $\mathbf{B}$ In vitro migration assay: the number of myosin $1 \mathrm{f}^{+/+}$neutrophils migrated from the upper chamber to the lower chamber was significantly higher compared to myosin $1 \mathrm{f}^{-/-}$ neutrophils. ${ }^{*} p=0.0463, n=3-4$ mice/group; each has 3 replicates. C Confocal immunofluorescent staining for MPO, which was counterstained with DAPI. (a) A representative brain section stained with cresol/violet is presented to show the three indicated regions where cell numbers were quantified. (b) Representative staining of $\mathrm{MPO}^{+}$(green) and DAPI (blue) at $72 \mathrm{~h}$ after stroke. (c) The bar graph represents the statistic results of the $\mathrm{MPO}^{+}$positive cells. Scale bar: $50 \mu \mathrm{m} .{ }^{* *} p=0.0024, n=5-6$ mice/group. D FACS quantification of infiltrating neutrophils in the ischemic brain. (a) The representative gating strategies of $\mathrm{CD}_{4} 5^{+}$, MiDMs (microglia-derived macrophages, $\mathrm{CD} 45^{\mathrm{Low}} \mathrm{CD} 11 \mathrm{~b}^{+}$), MoDM (monocytes-derived macrophages, $C D 45^{\mathrm{Hi}} \mathrm{CD} 11 \mathrm{~b}^{+}$), and neutrophils $\left(\mathrm{CD} 11 \mathrm{~b}^{+} \mathrm{Ly} 6 \mathrm{G}^{+}\right)$. (b) The cell numbers of total $\mathrm{CD} 45^{+}$leukocytes, neutrophils, MiDMs, and MoDMs. $\left(*^{*},{ }^{* *}, *^{* * *},{ }^{* * *}, p<0.05,0.01,0.001,0.0001\right.$, respectively. $N=3-5$ mice/group.)

tension generation $[8,39]$. Compared with myosin1e, myosin1f is specifically essential for neutrophil migration. Our in vivo studies indeed showed that myosin1f ${ }^{-/-}$mice had less neutrophils migrating into the ischemic hemisphere after stroke. Using adoptive transfer techniques, we further confirmed that myosin $1 \mathrm{f}$ in circulating neutrophils plays the key role, given that there is no myosin $1 f$ expression in the brain [8].

Our RT-PCR results showed that the myosin $1 f$ expression was significantly increased in peripheral neutrophils during the acute phase of ischemic stroke, indicating that the peripheral neutrophils may require a higher expression level of myosin $1 \mathrm{f}$ to migrate into the ischemic area. Previous studies have shown that myosin $1 \mathrm{f}$ is required for neutrophil migration in a 3D environment [12], as it regulates the deformation of the neutrophil nucleus to facilize the migration through physical barriers [12]. Therefore, the increases in myosin $1 \mathrm{f}$ expression in peripheral neutrophils are a response corresponding to the requirement of neutrophil migration into the ischemic brain.

Since the myosin1f KO in mice is a global knockout, we cannot exclude the possibility that the neutrophil migration was also influenced in the immune tissues, such as the spleen. Splenectomy has been reported to attenuate brain injury [40]. It served as a major reservoir for circulating immune cells, thus it is a crucial peripheral immune organ that mediates inflammation. Therefore, it is possible that myosin $1 \mathrm{f}$ gene $\mathrm{KO}$ results in fewer neutrophils emigrating from the spleen into the peripheral blood, leading to attenuated infiltration of neutrophils in the ischemic brain. In addition, neutrophils are known to mediate edema formation [41], and edema is a crucial step for brain injury after stroke [42]. We speculate that myosin $1 f \mathrm{KO}$ may protect against brain injury by attenuating brain edema by inhibiting neutrophil infiltration.

We are aware that our current study has some limitations. For instance, only young male adult animals were used, but gender has a significant influence on the stroke outcomes [43]. In addition, aging has a great impact on neuroinflammation [44]. Therefore, future experiments should be conducted by including female and aged animals.

\section{Conclusion}

We showed that the myosin $1 \mathrm{f}^{-/-}$mice had significantly smaller infarctions than the myosin $1 \mathrm{f}^{+/+}$mice, that peripheral neutrophils are the key cell types responsible for the effects of myosin $1 \mathrm{f}$ on brain injury, and that myo$\sin 1 \mathrm{f}$ determines neutrophil migration into the ischemic hemisphere. We therefore conclude that myosin $1 \mathrm{f}$ is an important factor for determining neutrophil migration into the ischemic brain, and it is a potential target for attenuating brain injury induced by stroke.

\section{Additional file}

Additional file 1: Figure S1. Representative flow cytometry analysis of neutrophil depletion. Single cells were obtained by gating with FSCheight vs. FSC-area, and then live/dead cells were gated by using Aqua to exclude dead cells. Peripheral blood neutrophils were first gated using FSC-A and SSC-A (for granulocytes) and then confirmed as CD45 $5^{+}$and $\mathrm{Ly}_{6 \mathrm{G}}{ }^{+}$cells. Figure S2. A. Experimental procedures. For neutrophil depletion, the recipient mice (CD45.2-) were IP injected with Ly6G antibodies on 1 day. The animals then received an adoptive transfer of purified neutrophils from WT type (CD45.2+) on 0 day. Then FACS analysis of the CD45.2 cells in the peripheral blood $36 \mathrm{~h}$ and $72 \mathrm{~h}$ after adoptive neutrophil transfer. B. $36 \mathrm{~h}$ after transfer, a group of cells still expressed CD45.2 but then stopped after $72 \mathrm{~h}$. Figure $\mathrm{S3}$. The comparison of relative gene expression for genes ICMA-1 (A) and MAC-1 (B) in blood neutrophils at 3 days after stroke between myosin $1 f^{+/+}(+/+)$and myosin $1 f^{-/-}(-/-)$ mice. $n=3$ /group; each has 3 replicates. There was no significant difference. Table S1. Cerebral blood flow (CBF) was measured before stroke onset, during stroke, and after reperfusion. Table S2. Primer sequences. (DOC $338 \mathrm{~kb}$ )

\section{Abbreviations}

APLAC: University Administrative Panel on Laboratory Animal Care; BBB: Brainblood barrier; CCA: Common carotid artery; FACS: Fluorescence-activated cell sorting; ICAM-1: Intracellular adhesion molecule 1; IF: Immunofluorescence staining; MAC-1: Macrophage-1antigen; MCAO: Middle cerebral artery occlusion; mNSS: A modified neurological severity score; MPO: Myeloperoxidase antibody; NIF: Neutrophil inhibitory factor; NK: Natural killer cells; PAF: Platelet-activating factor; RT-PCR: Reverse transcription polymerase chain reaction; VLA-4: Very-lateantigen-4; WT: Wild type

\section{Acknowledgements}

The authors wish to thank Ms. Felicia F. Beppu for language editing.

\section{Funding}

This work was supported by the National Institutes of Health Grant R01NS064236 to HZ; the Shanghai Eastern Scholar Program, the Shanghai Pujiang Program, NSFC General Program 81771739 to FW. 


\section{Availability of data and materials}

The datasets used in current study are available from the corresponding author on reasonable request.

\section{Authors' contributions}

YW, FW, and $\mathrm{HZ}$ conceptualized this work. YW, JHJ, WW, and FW conducted the experiment and analyze the data. YW and $\mathrm{HZ}$ drafted the manuscript. All authors approved the submission.

\section{Ethics approval}

Animal experimental protocols were approved by the Stanford University Administrative Panel on Laboratory Animal Care (APLAC). Experiments were performed in accordance with the ARRIVE guidelines and the National Institutes of Health $(\mathrm{NIH})$ Guidelines for the Care and Use of Laboratory Animals with local government approval.

\section{Consent for publication}

Not applicable.

\section{Competing interests}

The authors declare that they have no competing interests.

\section{Publisher's Note}

Springer Nature remains neutral with regard to jurisdictional claims in published maps and institutional affiliations.

\section{Author details}

'Department of Neurosurgery, Stanford University School of Medicine, 1201 Welch Road, MSLS Building, Room P306, Stanford, CA 94305, USA. ${ }^{2}$ Department of Plant Biology, Carnegie Institution for Science, Stanford, CA 94305, USA. ${ }^{3}$ Center for Microbiota and Immunological Diseases, Shanghai General Hospital, Shanghai Institute of Immunology, Shanghai Jiao Tong University School of Medicine, 280 South Chongqing Road, Building 5\#, Room 807\#, Shanghai 200025, China.

\section{Received: 7 January 2019 Accepted: 26 March 2019}

Published online: 10 April 2019

\section{References}

1. Moskowitz MA, Lo EH, ladecola C. The science of stroke: mechanisms in search of treatments. Neuron. 2010;67(2):181-98.

2. Neuhaus $A A$, et al. Neuroprotection in stroke: the importance of collaboration and reproducibility. Brain. 2017;140(8):2079-92.

3. Anrather J, ladecola C. Inflammation and stroke: an overview. Neurotherapeutics. 2016;13(4):661-70

4. Herz J, et al. Role of neutrophils in exacerbation of brain injury after focal cerebral ischemia in Hyperlipidemic mice. Stroke. 2015;46(10):2916-25.

5. Neumann J, et al. Very-late-antigen-4 (VLA-4)-mediated brain invasion by neutrophils leads to interactions with microglia, increased ischemic injury and impaired behavior in experimental stroke. Acta Neuropathol. 2015; 129(2):259-77.

6. Buck $\mathrm{BH}$, et al. Early neutrophilia is associated with volume of ischemic tissue in acute stroke. Stroke. 2008;39(2):355-60.

7. Richards TA, Cavalier-Smith T. Myosin domain evolution and the primary divergence of eukaryotes. Nature. 2005;436(7054):1113-8

8. Kim SV, et al. Modulation of cell adhesion and motility in the immune system by Myo1f. Science. 2006;314(5796):136-9.

9. Cordonnier MN, et al. Actin filaments and myosin I alpha cooperate with microtubules for the movement of lysosomes. Mol Biol Cell. 2001:12(12): 4013-29.

10. Bose $\mathrm{A}$, et al. Glucose transporter recycling in response to insulin is facilitated by myosin Myo1c. Nature. 2002;420(6917):821-4.

11. Tyska MJ, et al. Myosin-1a is critical for normal brush border structure and composition. Mol Biol Cell. 2005;16(5):2443-57.

12. Salvermoser $M$, et al. Myosin $1 \mathrm{f}$ is specifically required for neutrophil migration in 3D environments during acute inflammation. Blood. 2018; 131(17):1887-98

13. Kilkenny $C$, et al. Animal research: reporting in vivo experiments: the ARRIVE guidelines. Br J Pharmacol. 2010;160(7):1577-9.

14. Becker KJ, Buckwalter M. Stroke, inflammation and the immune response: Dawn of a new era. Neurotherapeutics. 2016;13(4):659-60.
15. Schneider CA, Rasband WS, Eliceiri KW. NIH image to ImageJ: 25 years of image analysis. Nat Methods. 2012;9(7):671-5.

16. Takahashi T, Steinberg GK, Zhao H. Lithium treatment reduces brain injury induced by focal ischemia with partial reperfusion and the protective mechanisms dispute the importance of akt activity. Aging Dis. 2012;3(3):226-33.

17. Li Y, et al. Intrastriatal transplantation of bone marrow nonhematopoietic cells improves functional recovery after stroke in adult mice. J Cereb Blood Flow Metab. 2000;20(9):1311-9.

18. Wang F, et al. Inhibition of T cell receptor signaling by cholesterol sulfate, a naturally occurring derivative of membrane cholesterol. Nat Immunol. 2016; 17(7):844-50.

19. Ren $\mathrm{H}$, et al. Selective NLRP3 (pyrin domain-containing protein 3) Inflammasome inhibitor reduces brain injury after intracerebral hemorrhage. Stroke. 2018;49(1):184-92.

20. Faul F, et al. G*power 3: a flexible statistical power analysis program for the social, behavioral, and biomedical sciences. Behav Res Methods. 2007;39(2):175-91.

21. Jing $L$, et al. Upregulation of ICAM-1 in diabetic rats after transient forebrain ischemia and reperfusion injury. J Inflamm (Lond). 2014;11(1):35.

22. Lindsberg PJ, et al. Endothelial ICAM-1 expression associated with inflammatory cell response in human ischemic stroke. Circulation. 1996; 94(5):939-45.

23. Zhang RL, et al. Anti-intercellular adhesion molecule-1 antibody reduces ischemic cell damage after transient but not permanent middle cerebral artery occlusion in the Wistar rat. Stroke. 1995;26(8):1438-42 discussion 1443.

24. Jin R, Yang G, Li G. Inflammatory mechanisms in ischemic stroke: role of inflammatory cells. J Leukoc Biol. 2010;87(5):779-89.

25. Yilmaz G, Granger DN. Leukocyte recruitment and ischemic brain injury. NeuroMolecular Med. 2010;12(2):193-204.

26. Segel GB, Halterman MW, Lichtman MA. The paradox of the neutrophil's role in tissue injury. J Leukoc Biol. 2011;89(3):359-72.

27. Cuartero Ml, et al. N2 neutrophils, novel players in brain inflammation after stroke: modulation by the PPARY agonist rosiglitazone. Stroke. 2013;44(12): 3498-508.

28. Morrison H, McKee D, Ritter L. Systemic neutrophil activation in a mouse model of ischemic stroke and reperfusion. Biol Res Nurs. 2011:13(2):154-63.

29. Adeoye $\mathrm{O}$, et al. Peripheral monocyte count is associated with case fatality after intracerebral hemorrhage. J Stroke Cerebrovasc Dis. 2014;23(2):e107-11.

30. Moxon-Emre I, Schlichter LC. Neutrophil depletion reduces blood-brain barrier breakdown, axon injury, and inflammation after intracerebral hemorrhage. J Neuropathol Exp Neurol. 2011;70(3):218-35.

31. Kolaczkowska E, Kubes P. Neutrophil recruitment and function in health and inflammation. Nat Rev Immunol. 2013;13(3):159-75.

32. Jickling GC, et al. Targeting neutrophils in ischemic stroke: translational insights from experimental studies. J Cereb Blood Flow Metab. 2015;35(6):888-901.

33. Lindsberg PJ, et al. Antagonism of neutrophil adherence in the deteriorating stroke model in rabbits. J Neurosurg. 1995:82(2):269-77.

34. Kitagawa K, et al. Deficiency of intercellular adhesion molecule 1 attenuates microcirculatory disturbance and infarction size in focal cerebral ischemia. J Cereb Blood Flow Metab. 1998;18(12):1336-45.

35. Jiang $N$, et al. Neutrophil inhibitory factor is neuroprotective after focal ischemia in rats. Ann Neurol. 1995;38(6):935-42.

36. Zhang $L$, et al. Effects of a selective CD11b/CD18 antagonist and recombinant human tissue plasminogen activator treatment alone and in combination in a rat embolic model of stroke. Stroke. 2003;34(7):1790-5.

37. McIntosh BB, Ostap EM. Myosin-I molecular motors at a glance. J Cell Sci. 2016;129(14):2689-95.

38. Swanson JA, et al. A contractile activity that closes phagosomes in macrophages. J Cell Sci. 1999;112(Pt 3):307-16.

39. Gauthier NC, Masters TA, Sheetz MP. Mechanical feedback between membrane tension and dynamics. Trends Cell Biol. 2012;22(10):527-35.

40. Ajmo $C T$, et al. The spleen contributes to stroke-induced neurodegeneration. J Neurosci Res. 2008;86(10):2227-34.

41. Kenne E, et al. Neutrophil depletion reduces edema formation and tissue loss following traumatic brain injury in mice. J Neuroinflammation. 2012;9:17.

42. Battey TW, et al. Brain edema predicts outcome after nonlacunar ischemic stroke. Stroke. 2014:45(12):3643-8.

43. Roy-O'Reilly M, McCullough LD. Sex differences in stroke: the contribution of coagulation. Exp Neurol. 2014;259:16-27.

44. Fonarow GC, et al. Age-related differences in characteristics, performance measures, treatment trends, and outcomes in patients with ischemic stroke. Circulation. 2010;121:879-91. 